\title{
Nitrogênio proveniente da adubação nitrogenada e de resíduos culturais na nutrição da cana-planta
}

\author{
André Cesar Vitti(1), Henrique Coutinho Junqueira Franco(2), Paulo Cesar Ocheuze Trivelin ${ }^{(3)}$, \\ Danilo Alves Ferreira ${ }^{(3)}$, Rafael Otto(3), Caio Fortes ${ }^{(3)}$ e Carlos Eduardo Faroni( ${ }^{(4)}$
}

(1)Agência Paulista de Tecnologia dos Agronegócios (APTA), Polo Regional Centro-Sul, Rodovia SP-127, Km 30, Caixa Postal 28, CEP 13400-970 Piracicaba, SP. E-mail: acvitti@apta.sp.gov.br (2)Centro de Pesquisa em Energia e Materiais, Laboratório Nacional de Ciência e Tecnologia do Bioetanol, Rua Giuseppe Máximo Scolfaro, № 10.000, Bairro Guará, Distrito de Barão Geraldo, CEP 13083-970 Campinas, SP. E-mail: henrique.franco@bioetanol.org.br (3)Universidade de São Paulo, Centro de Energia Nuclear na Agricultura, Caixa Postal 96, CEP 13400-970 Piracicaba, SP. E-mail: pcotrive@cena.usp.br, danilo.alves.ferreira@usp.br, rotto@esalq.usp.br, cfortes@cena.usp.br ${ }^{(4)}$ Centro de Tecnologia Canavieira, Caixa Postal 162, CEP 13400-970 Piracicaba, SP. E-mail: cfaroni@ctc.com.br

Resumo - O objetivo deste trabalho foi avaliar a recuperação, pela cana-planta, do nitrogênio $\left({ }^{15} \mathrm{~N}\right)$ proveniente da ureia e de resíduos culturais da palhada e do sistema radicular da cultura da cana (Saccharum spp.) incorporados ao solo. O experimento foi instalado na safra de 2005/2006, com a cultivar SP81-3250. No plantio, foram instaladas microparcelas de $1,5 \times 2 \mathrm{~m}$, em que aplicaram-se doses equivalentes a $80 \mathrm{~kg} \mathrm{ha}^{-1} \mathrm{de} \mathrm{N}$ (ureia com $5,05 \%$ de ${ }^{15} \mathrm{~N}$ ) e $14 \mathrm{Mg} \mathrm{ha}^{-1}$ de resíduos culturais, dos quais $9 \mathrm{Mg} \mathrm{ha}^{-1}$ de palhada (PA) e $5 \mathrm{Mg} \mathrm{ha}^{-1} \mathrm{de}$ sistema radicular (SR), marcados com ${ }^{15} \mathrm{~N}\left(1,07\right.$ e $0,81 \%$ de ${ }^{15} \mathrm{~N}$, respectivamente). Durante o ciclo da cultura, determinou-se o acúmulo total de $\mathrm{N}$ da planta. Embora o aproveitamento do $\mathrm{N}$ oriundo da mineralização dos resíduos culturais (PA e SR) pela parte aérea tenha aumentado expressivamente com o tempo, esta fonte pouco contribuiu para a nutrição da cultura. A recuperação pela cana-planta de ${ }^{15} \mathrm{~N}$-ureia, ${ }^{15} \mathrm{~N}-\mathrm{PA}$ e ${ }^{15} \mathrm{~N}-\mathrm{SR}$ foi de $30,3 \pm 3,7 \%, 13,9 \pm 4,5 \%$ e $6,4 \pm 0,9 \%$ respectivamente, o que representa $15,9,4,7$ e $1,4 \%$ do nitrogênio total acumulado pela parte aérea da cultura.

Termos para indexação: Saccharum, cana-de-açúcar, ${ }^{15} \mathrm{~N}$, raízes, ureia.

\section{Nitrogen derived from fertilization and straw for plant cane nutrition}

\begin{abstract}
The objective of this work was to evaluate the recovery, by plant cane, of the nitrogen $\left({ }^{15} \mathrm{~N}\right)$ from urea and from sugarcane (Saccharum spp.) crop residues - straw and root system - incorporated into the soil. The experiment was settled in 2005/2006 with the sugarcane cultivar SP81 3250. At planting, microplots of $2 \mathrm{~m}$ length and $1.5 \mathrm{~m}$ width were installed, and $\mathrm{N}$ applications were done with $80 \mathrm{~kg} \mathrm{ha}^{-1} \mathrm{~N}$ (urea with $5.05 \%$ in ${ }^{15} \mathrm{~N}$ atoms) and $14 \mathrm{Mg} \mathrm{ha}^{-1}$ crop residues $-9 \mathrm{Mg} \mathrm{ha}^{-1}$ of sugarcane straw (SS) and $5 \mathrm{Mg} \mathrm{ha}^{-1}$ of root system (RS), labeled with ${ }^{15} \mathrm{~N}$ (1.07 and $0.81 \%$ in ${ }^{15} \mathrm{~N}$ atoms, respectively). The total $\mathrm{N}$ accumulation by plants was determined during the crop cycle. Although the $\mathrm{N}$ use by shoot from crop residue mineralization (PA and SR) increased significantly over time, this source hardly contributed to crop nutrition. The recovery of the ${ }^{15} \mathrm{~N}$-urea, ${ }^{15} \mathrm{~N}-\mathrm{SS}$ and ${ }^{15} \mathrm{~N}-\mathrm{RS}$ by plant cane was $30.3 \pm 3.7 \%, 13.9 \pm 4.5 \%$ and $6.4 \pm 0.9 \%$, respectively, representing 15.9 , 4.7 and $1.4 \%$ of total nitrogen uptake by shoot.
\end{abstract}

Index terms: Saccharum, sugarcane, ${ }^{15} \mathrm{~N}$, roots, urea.

\section{Introdução}

$\mathrm{Na}$ cultura da cana-de-açúcar, a realização da colheita sem queima deixa sobre a superfície do solo quantidade considerável de resíduos vegetais, de 10 a $30 \mathrm{Mg} \mathrm{ha}^{-1}$ de massa de matéria seca (Trivelin et al., 1995; Vitti et al., 2007). A manutenção da palhada sem queima traz uma série de vantagens ao sistema solo-planta, como o aumento do estoque de carbono do solo (Galdos et al., 2009). A quantidade de palhada depositada na superfície do solo depende da cultivar, produtividade, época de corte, entre outros, e pode conter de 80 a $200 \mathrm{~kg} \mathrm{ha}^{-1}$ de $\mathrm{N}$ (Franco et al.,
2007). Em áreas de reforma do canavial sem queima, a quantidade de resíduos orgânicos é ainda maior, uma vez que além da palhada há o sistema radicular e a parte aérea da soqueira antiga, o que aumenta a quantidade de material orgânico e nutrientes que serão incorporados ao solo (Vitti et al., 2007).

Fatores ambientais, como temperatura, umidade e aeração, e fatores químicos que dependem, principalmente, da qualidade dos resíduos culturais, especialmente da relação $\mathrm{C}: \mathrm{N}$, teores de lignina, celulose, hemicelulose e polifenóis, influenciam a mineralização desse resíduo cultural e, portanto, a disponibilidade de nutrientes às plantas (Gupta \&

Pesq. agropec. bras., Brasília, v.46, n.3, p.287-293, mar. 2011 
Singh, 1981; Ng Kee Kwong et al., 1987; Meier et al., 2006). As quantidades de $\mathrm{N}$ liberadas desses resíduos durante o ciclo seguinte da cultura de cana-de-açúcar podem variar de 3 a 30\% (Vitti, 2008; Vitti et al., 2010). Estudos mostraram que a utilização de $\mathrm{N}$ de resíduos vegetais $\left({ }^{15} \mathrm{~N}\right)$, pela cana-de-açúcar, variou de 5 a 20\% (Ng Kee Kwong et al., 1987; Gava et al., 2003; Bologna-Campbell, 2007). Essa variação, segundo os autores, depende da qualidade do resíduo, pois quando a concentração de $\mathrm{N}$ é maior que $20 \mathrm{~g} \mathrm{~kg}^{-1}$ e a relação $\mathrm{C}: \mathrm{N}$ menor que 25 , a mineralização dos resíduos é mais rápida e, consequentemente, maior será $\mathrm{o}$ aproveitamento de nutrientes pela cultura cultivada na sequência.

$\mathrm{O}$ balanço do $\mathrm{N}$ marcado com o isótopo ${ }^{15} \mathrm{~N}$, proveniente da palhada de cana-de-açúcar, realizado por Vitti (2003) indicou que, depois de 12 meses, dos $62 \mathrm{~kg} \mathrm{ha}^{-1}$ de $\mathrm{N}$ presente na palhada, apenas $2,4 \mathrm{~kg} \mathrm{ha}^{-1}$ de foram acumulados pela parte aérea da soqueira subsequente (parte aérea e sistema radicular), o que representou cerca de $4 \%$ do $\mathrm{N}$ desse resíduo cultural. O baixo aproveitamento do N-palhada pode ser explicado pela decomposição lenta do resíduo no primeiro ano (Vitti et al., 2008), principalmente em razão da alta relação $\mathrm{C}: \mathrm{N}$ inicial da palha de cana-de-açúcar, que favorece a imobilização do $\mathrm{N}$ pelos microrganismos do solo em vez da mineralização (Jingguo \& Bakken, 1997). Ng Kee Kwong et al. (1987) verificaram que a utilização do $\mathrm{N}$ da palha pela cana-de-açúcar foi de 10,6 a 13,3. Porém, ao incorporar os resíduos culturais ao solo, para simular a reforma do canavial, Bologna-Campbell (2007) obteve uma recuperação ligeiramente maior, da ordem de $20 \%$.

Assim, pressupõe-se que, durante a reforma de áreas com cana-de-açúcar, a quantidade de material orgânico e $\mathrm{N}$ disponíveis à mineralização é maior do que em áreas de soqueira, o que pode ser uma importante fonte de $\mathrm{N}$ para o novo ciclo da cana-planta.

O objetivo deste trabalho foi avaliar a utilização, pela cana-planta, do nitrogênio $\left({ }^{15} \mathrm{~N}\right)$ proveniente de resíduos culturais da palhada e do sistema radicular, incorporados ao solo na implantação da cultura, e do ${ }^{15} \mathrm{~N}$-ureia aplicado no sulco de plantio.

\section{Material e Métodos}

O experimento foi desenvolvido em área comercial de cana-de-açúcar da Usina São Martinho, no
Município de Pradópolis, SP, $21^{\circ} 15^{\prime} \mathrm{S}, 48^{\circ} 18^{\prime} \mathrm{W}$, à altitude de $580 \mathrm{~m}$, com tipo climático Aw, tropical de savana, segundo a classificação de Köppen. A área, com declividade de 5-10\%, é de Latossolo Vermelho eutrófico (Santos et al., 2006), com histórico de sete anos consecutivos de colheita mecânica, sem queima (cana-crua) da cultura. A análise química do solo, realizada antes da instalação do experimento, em janeiro de 2005, apresentou os seguintes valores às profundidades de $0-25$ e $25-50 \mathrm{~cm}$, respectivamente: 5,2 e 5,6 (pH em $\left.\mathrm{CaCl}_{2}\right) ; 31$ e $18 \mathrm{~g} \mathrm{dm}^{-3}$ (MO); 42 e $12 \mathrm{mg} \mathrm{dm}^{-3}$ (P resina); 3,1 e 0,5 $\mathrm{mmol}_{\mathrm{c}} \mathrm{dm}^{-3}(\mathrm{~K}) ; 31$ e $24 \mathrm{mmol}_{\mathrm{c}} \mathrm{dm}^{-3}(\mathrm{Ca}) ; 9$ e $6 \mathrm{mmol}_{\mathrm{c}} \mathrm{dm}^{-3}(\mathrm{Mg}) ; 34 \mathrm{e}$ $22 \mathrm{mmol}_{\mathrm{c}} \mathrm{dm}^{-3}(\mathrm{H}+\mathrm{Al}) ; 1,0$ e 1,0 $\mathrm{mmol}_{\mathrm{c}} \mathrm{dm}^{-3}(\mathrm{Al}) ; 74,4$ e $53,0 \mathrm{mmol}_{\mathrm{c}} \mathrm{dm}^{-3}$ (CTC); e 56 e $58 \%$ (saturação por bases).

Antes da instalação do experimento, a área foi preparada pelo método do cultivo mínimo, com dessecação da soqueira antiga pelo glifosato, na dose de $1.920 \mathrm{~g} \mathrm{ha}^{-1}$ de i.a., aplicados à área total, em janeiro de 2005. Em seguida, foi realizada a subsolagem, com subsolador com hastes de $0,8 \mathrm{~m}$ de comprimento, à profundidade efetiva de operação, em torno de $0,4 \mathrm{~m}$. Após a subsolagem, foi realizada a abertura dos sulcos com profundidade média de $0,35 \mathrm{~m}$, e o plantio foi realizado entre 2 a 7 de março de 2005 .

Utilizou-se o delineamento experimental de blocos ao acaso, com quatro repetições. No plantio, aplicaram-se $80 \mathrm{~kg} \mathrm{ha}^{-1}$ de N, na forma de ureia, no fundo do sulco, em todas as parcelas. No interior de cada parcela, foram construídas três microparcelas com dimensão de $2 \mathrm{~m}$ de comprimento e 1,5 $\mathrm{m}$ de largura, que receberam a ureia e os resíduos culturais, palhada e sistema radicular, marcados em ${ }^{15} \mathrm{~N}$. Em cada microparcela, das três fontes de $\mathrm{N}$, somente uma era marcada em ${ }^{15} \mathrm{~N}$; as demais eram substituídas pela mesma fonte de $\mathrm{N}$, porém com composição isotópica natural de $0,366 \%$ em átomos de ${ }^{15} \mathrm{~N}$. Assim, foi possível avaliar a contribuição do $\mathrm{N}$ de cada fonte (ureia, palhada e sistema radicular) no acúmulo de $\mathrm{N}$ total da cana-planta. A quantidade usada dos resíduos culturais palhada (PA) e sistema radicular (SR) foi equivalente a 9 e $5 \mathrm{Mg} \mathrm{ha}^{-1}$, respectivamente. $\mathrm{O}$ teor médio de $\mathrm{N}$ total e ${ }^{15} \mathrm{~N}$ da palhada foi de $0,57 \pm 0,01 \%$ e $1,07 \pm 0,01 \%$, respectivamente, com aporte de $51,5 \pm 1,1 \mathrm{~kg} \mathrm{ha}^{-1}$ de $\mathrm{N}$. No sistema radicular, esses valores foram de $0,66 \pm 0,03 \%$ e $0,81 \pm 0,01 \%$, com aporte de $33,0 \pm 1,8 \mathrm{~kg} \mathrm{ha}{ }^{-1}$ de N. A palhada- ${ }^{15} \mathrm{~N}$, composta por folhas secas e ponteiros, e o sistema 
radicular- ${ }^{15} \mathrm{~N}$ provieram de soqueira da cultivar RB85-5536, produzida em experimento prévio na safra 2004/2005. A técnica de marcação das plantas de cana-de-açúcar com ${ }^{15} \mathrm{~N}$ utilizou a aplicação foliar de solução de ureia, em baixa dose e alto enriquecimento de ${ }^{15} \mathrm{~N}, 35 \%$ em átomos de ${ }^{15} \mathrm{~N}$ (Faroni et al., 2007).

O fertilizante nitrogenado foi aplicado no sulco de plantio e incorporado ao solo antes da distribuição dos colmos, em março de 2005. Os resíduos culturais das raízes e da palhada foram parcialmente incorporados ao solo, para simular o preparo mínimo do solo antes da implantação do experimento. As parcelas experimentais eram compostas por 48 linhas (sulcos) de $15 \mathrm{~m}$ de comprimento, espaçadas entre si por 1,5 m. No plantio, foram aplicados, no fundo dos sulcos, em todas as parcelas, o cloreto de potássio e o superfosfato simples, que forneceram $120 \mathrm{~kg} \mathrm{ha}^{-1}$ de $\mathrm{K}_{2} \mathrm{O}$ e $120 \mathrm{~kg} \mathrm{ha}^{-1}$ de $\mathrm{P}_{2} \mathrm{O}_{5}$, respectivamente. No plantio, as mudas da cultivar SP81-3250 apresentavam cerca de 20 gemas por metro linear de sulco. As mudas de cana, depositadas no fundo do sulco, foram cortadas em toletes com 2 a 3 gemas. Durante o ciclo da cana-planta, foram mensuradas as precipitações pluviais e a temperatura média, em estação meteorológica instalada a cerca de 5 $\mathrm{km}$ do experimento (Figura 1). Os dados históricos de precipitação pluvial foram obtidos do mesmo local.

Em dezembro de 2005, e em fevereiro, abril e agosto de 2006, foi realizada, em cada parcela, a coleta de toda a parte aérea (colmos, folhas secas e ponteiros) de $2 \mathrm{~m}$ da linha de plantio. Em seguida, obteve-se a massa de matéria verde mediante pesagem no campo. Toda a parte aérea foi triturada em picadora de forragem, tendo-se obtido uma subamostra, que foi seca em estufa a $65^{\circ} \mathrm{C}$ durante 72 horas, para obtenção da massa de matéria seca. Nessas amostras, realizou-se a análise de $\mathrm{N}$ total (Malavolta et al., 1997), e calculou-se o acúmulo de $\mathrm{N}$ total $\left(\mathrm{kg} \mathrm{ha}^{-1}\right)$ pela cultura, em cada época de amostragem.

Simultaneamente, nas mesmas épocas de amostragem de parte aérea, foram colhidas amostras de folhas +3 (terceira folha, de cima para baixo, com a aurícula perfeitamente visível) em todas as microparcelas, exceto em dezembro na microparcela com ureia marcada. O padrão de amostragem utilizado foi o mesmo adotado por Franco et al. (2011). Nessas amostragens, coletaram-se, no centro de cada microparcela, duas folhas +3 , que constituíram uma amostra. Adicionalmente, coletaram-se duas folhas nas linhas adjacentes, em posições contíguas às microparcelas que, juntas, constituíram outra amostra. As amostras foram secas em estufa ventilada a $65^{\circ} \mathrm{C}$, e moídas em moinho tipo Willey. A abundância de ${ }^{15} \mathrm{~N}$ (percentagem de átomos de ${ }^{15} \mathrm{~N}$ ) foi determinada em espectrômetro de massas modelo 20/20 ANCA/SL, (SerCon Ltda., Cheschire, UK). Franco et al. (2011) observaram semelhança entre os valores de abundância

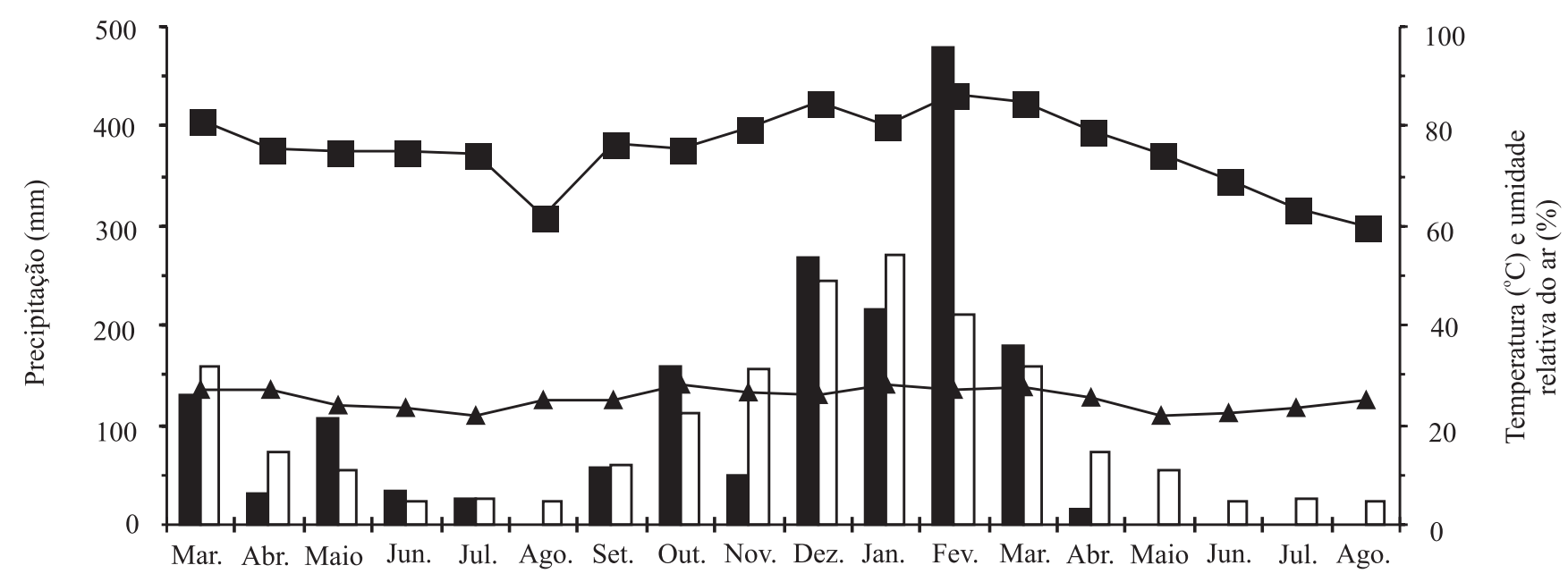

Mês Precipitação $\square$ Precipitação média Tmédia UR

Figura 1. Precipitação pluvial, mensal e histórica (1968-2008), e temperatura média do ar durante o desenvolvimento do experimento de março de 2005 a agosto de 2006. 
de ${ }^{15} \mathrm{~N}$ da folha +3 e da planta toda de cana-de-açúcar, e concluíram que a folha +3 pode ser usada para estimar a recuperação de ${ }^{15} \mathrm{~N}$ de fontes marcadas, durante $\mathrm{o}$ ciclo de crescimento. Assim, os dados de acúmulo de $\mathrm{N}$ na parte aérea obtidos em amostras coletadas fora da microparcela, e da abundância isotópica da folha +3 obtidos dentro da microparcela, foram usados para estimar, em cada estádio de crescimento, o nitrogênio na parte aérea derivado do fertilizante (NPPF), da palhada (NPPP) e do sistema radicular (NPPSR), pelo método da diluição isotópica do ${ }^{15} \mathrm{~N}$.

A colheita da cana-planta foi realizada em agosto de 2006. Nessa oportunidade, uma testemunha sem componente marcado e a parte aérea das plantas das microparcelas foram colhidas manualmente, no centro das microparcelas $(1 \mathrm{~m})$ e em posições contíguas nas linhas adjacentes à microparcela. Amostras de folhas secas, ponteiros e colmos foram retiradas, e a massa de matéria verde foi determinada. Todo o material foi triturado em picadora mecânica de forragem. Depois da moagem e homogeneização de cada amostra úmida, retirou-se uma subamostra, que foi seca em estufa (72 horas a $65{ }^{\circ} \mathrm{C}$ ), e cuja umidade foi determinada. $\mathrm{O}$ material seco foi moído em moinho tipo Willey e usado nas determinações de N-total (Malavolta et al., 1997) e de abundância de ${ }^{15} \mathrm{~N}$ (\% em átomos de $\left.{ }^{15} \mathrm{~N}\right)$, em espectrômetro de massa modelo 20/20 ANCA/SL, (SerCon Ltd.., Cheschire, UK).

$\mathrm{O}$ nitrogênio na planta proveniente de fonte marcada (Nppfm) e a recuperação do $\mathrm{N}(\mathrm{R})$ pela parte aérea de cada componente marcado foram calculados por meio das equações: $\mathrm{Nppfm}=[(\mathrm{A}-\mathrm{C}) /(\mathrm{B}-\mathrm{C})] \mathrm{NT}$ e $R(\%)=(N p p f m / N a f) 100$, em que: Nppfm é a quantidade de $\mathrm{N}$ na planta proveniente da fonte marcada $\left({ }^{15} \mathrm{~N}\right.$-ureia, ${ }^{15} \mathrm{~N}$-palhada ou ${ }^{15} \mathrm{~N}$-sistema radicular); A é a abundância de $\mathrm{N}$ (\% de átomos) da planta; $\mathrm{B}$ é a abundância de ${ }^{15} \mathrm{~N}$ das fontes marcadas $\left(5,04,1,07\right.$ e $0,81 \%$ de átomos de ${ }^{15} \mathrm{~N}$-ureia, ${ }^{15} \mathrm{~N}$-palhada e ${ }^{15} \mathrm{~N}$-sistema radicular, respectivamente); C é a abundância natural de ${ }^{15} \mathrm{~N}(0,366 \%$ de átomos); NT é o $\mathrm{N}$ total na planta $\left(\mathrm{kg} \mathrm{ha}^{-1}\right)$; R é a recuperação percentual de ${ }^{15} \mathrm{~N}$-ureia e dos ${ }^{15} \mathrm{~N}$-resíduos vegetais (palhada e sistema radicular), pela parte aérea da cana-planta; e Naf é a quantidade de $\mathrm{N}$ das fontes aplicadas $\left(\mathrm{kg} \mathrm{ha}^{-1}\right)$.

Os resultados foram analisados pela análise de variância e comparados pelo teste de Tukey, a 5\% de probabilidade, dentro de cada época de amostragem. $\mathrm{Na}$ colheita final das microparcelas com marcação isotópica, foram calculados os desvios-padrão das médias dos tratamentos (sistema radicular, palhada e ureia), dentro de cada compartimento de planta.

\section{Resultados e Discussão}

A maior recuperação de $\mathrm{N}$-ureia foi observada no início do ciclo da cultura, ao passo que, nos resíduos culturais (palhada e sistema radicular), ela ocorreu mais próximo da colheita da cana-planta (Tabela 1). Os valores de recuperação de $\mathrm{N}$-ureia foram maiores do que os encontrados por $\mathrm{Ng}$ Kwong \& Deville (1994) e Vitti (2003). Isso, provavelmente, ocorreu em razão de a recuperação ter sido calculada a partir de uma estimativa. Exemplo disso foi que, na colheita de agosto de 2006, a recuperação obtida pela estimativa $(\mathrm{F}+3)$ foi de $41,3 \%$, enquanto a recuperação real, obtida diretamente pela colheita da parte área nas microparcelas, foi de 30,3\%, valor normalmente encontrado na literatura (Tabela 2).

Para os resultados de $\mathrm{N}$ proveniente do fertilizante (NPPF), os maiores valores ocorreram na fase de máximo crescimento da cultura, em dezembro-janeiro, e estão associados a condições climáticas mais favoráveis de pluviosidade, temperatura e dias longos.

Tabela 1. Quantidades de N na planta provenientes da ureia (NPPF), da palhada (NPPP) e do sistema radicular (NPPSR), e recuperação $(\mathrm{R})$ do $\mathrm{N}$ de cada uma dessas fontes, ao longo do ciclo da cana-planta ${ }^{(1)}$.

\begin{tabular}{|c|c|c|c|c|c|c|c|c|c|c|}
\hline \multirow[t]{3}{*}{ Amostragem } & \multicolumn{3}{|c|}{ Ureia } & \multicolumn{3}{|c|}{ Palhada } & \multicolumn{3}{|c|}{ Sistema radicular } & \multirow{3}{*}{$\begin{array}{l}\text { NTPA }^{(2)} \\
\left(\mathrm{kg} \mathrm{ha}^{-1}\right)\end{array}$} \\
\hline & \multicolumn{2}{|c|}{ NPPF } & \multirow{2}{*}{$\begin{array}{c}\mathrm{R} \\
(\%)\end{array}$} & \multicolumn{2}{|c|}{ NPPP } & \multirow{2}{*}{$\begin{array}{c}\mathrm{R} \\
(\%)\end{array}$} & \multicolumn{2}{|c|}{ NPPSR } & \multirow{2}{*}{$\begin{array}{c}\mathrm{R} \\
(\%)\end{array}$} & \\
\hline & $(\%)$ & $\left(\mathrm{kg} \mathrm{ha}^{-1}\right)$ & & $(\%)$ & $\left(\mathrm{kg} \mathrm{ha}^{-1}\right)$ & & $(\%)$ & $\left(\mathrm{kg} \mathrm{ha}^{-1}\right)$ & & \\
\hline Dezembro & - & - & - & $1,2 \mathrm{Aa}$ & $2,4 \mathrm{Aa}$ & 5,0 & $0,4 \mathrm{Aa}$ & $0,8 \mathrm{Aa}$ & 2,4 & $202 \pm 13$ \\
\hline Fevereiro & $21,4 \mathrm{Aa}$ & $43,4 \mathrm{Aa}$ & 51,8 & $0,6 \mathrm{Ab}$ & $1,2 \mathrm{Ab}$ & 2,4 & $0,7 \mathrm{Ab}$ & $1,4 \mathrm{Ab}$ & 3,9 & $203 \pm 11$ \\
\hline Abril & $15,8 \mathrm{Ba}$ & $41,4 \mathrm{Aa}$ & 51,7 & $1,0 \mathrm{Ab}$ & $2,6 \mathrm{Ab}$ & 4,9 & $1,0 \mathrm{Ab}$ & $2,6 \mathrm{Ab}$ & 7,6 & $262 \pm 6$ \\
\hline Agosto & $13,9 \mathrm{Ba}$ & $33,0 \mathrm{Ba}$ & 41,3 & $1,4 \mathrm{Ab}$ & $3,1 \mathrm{Ab}$ & 6,0 & $0,9 \mathrm{Ab}$ & $2,2 \mathrm{Ab}$ & 6,5 & $237 \pm 28$ \\
\hline
\end{tabular}

${ }^{(1)}$ Médias seguidas por letras iguais, maiúsculas entre as fontes de N, durante as épocas de amostragem, e minúsculas entre as fontes dentro da mesma época de amostragem, não diferem pelo teste de Tukey, a 5\% de probabilidade. ${ }^{(2)}$ Média \pm desvio-padrão $(\mathrm{n}=4)$ do nitrogênio total da parte aérea. 
A partir desse estádio, ocorreu tendência de redução no valor de NPPF. Não se pode ignorar possíveis perdas ou translocação de $\mathrm{N}$ da parte aérea para o sistema radicular, principalmente entre os meses de abril e agosto de 2006, na fase de maturação do canavial, em que a redução do N-total no período foi de $25 \mathrm{~kg} \mathrm{ha}^{-1} \mathrm{de}$ $\mathrm{N}$, cerca de $10 \%$ do $\mathrm{N}$ total acumulado na parte aérea.

A redução na quantidade recuperada de $\mathrm{N}$-fertilizante, na fase de maturação da cana-planta, foi semelhante à observada com o N-total da parte aérea. Nesse período, houve diminuição de $8,4 \mathrm{~kg} \mathrm{ha}^{-1}$ de N proveniente do ${ }^{15} \mathrm{~N}$-ureia (redução de $10,4 \%$ no valor total da recuperação). Ng Kee Kwong \& Deville (1994) obtiveram perdas de 10 a $20 \mathrm{~kg} \mathrm{ha}^{-1}$ de $\mathrm{N}$-fertilizante, pela parte aérea da cana-de-açúcar, o que equivale a perdas de até $100 \mathrm{~kg} \mathrm{ha}^{-1}$ de $\mathrm{N}$, considerando-se o $\mathrm{N}$ total acumulado pela planta toda. Valores semelhantes de perdas pela parte aérea das plantas de cana-de-açúcar foram obtidos por Trivelin et al. (2002). Segundo Neirynck et al. (2005), a perda pela parte aérea (PA) dos vegetais pode representar cerca de $14 \%$ do fluxo líquido das emissões gasosas de $\mathrm{NH}_{3}$. Entretanto, parte dessa amônia emitida para a atmosfera é reabsorvida pela folhagem da própria planta ou de outra vegetação (Fenili et al., 2007; Martha Júnior et al., 2009). O N proveniente do fertilizante, por estar prontamente disponível às plantas, foi absorvido em maior quantidade no início e diminuiu ao longo do ciclo da cultura. Portanto, a chance de terem ocorrido perdas de $\mathrm{N}$-fertilizante pela parte aérea foi grande nessa fase. No entanto, pelo fato de o $\mathrm{N}$ dos resíduos culturais ser disponibilizado lentamente, em consequência da elevada relação $\mathrm{C}: \mathrm{N}$, a ocorrência de perdas de $\mathrm{N}$, pela parte aérea dessas fontes, é provavelmente menor, ou até, muitas vezes, desprezível.

Em razão das reduções nas quantidades de $\mathrm{N}$ na parte aérea, entre os períodos de desenvolvimento da cultura, há risco de se subestimar a recuperação de $\mathrm{N}$-fertilizante, quando o balanço de $\mathrm{N}$-fertilizante é realizado apenas no final do ciclo da cultura (colheita). Nesse sentido, Franco et al. (2011) observaram que o NPPF foi de $40 \%$, no início do ciclo da cana-planta, e diminuiu para $10 \%$ no final do ciclo, em consequência do aumento da participação de outras fontes na nutrição nitrogenada, durante o desenvolvimento da cultura. Segundo Vitti (2003), ao se levar em consideração o período em que ocorreu a maior recuperação de $\mathrm{N}$-fertilizante, e não o final do ciclo, a recuperação de $\mathrm{N}$-fertilizante seria de 33 e $47 \%$ do ${ }^{15} \mathrm{~N}^{-\mathrm{NH}_{4}}{ }^{+}$e ${ }^{15} \mathrm{~N}_{-} \mathrm{NO}_{3}{ }^{-}$, respectivamente, e não os 19 e $27 \%$ obtidos na colheita. Nesse caso, a recuperação total de $\mathrm{N}$-fertilizante no sistema solo-planta passaria a ter valores maiores, próximos a $80 \%$, e não apenas os $63 \%$ recuperados, obtidos na colheita.

$\mathrm{O}$ nitrogênio proveniente do $\mathrm{N}$ mineralizado da palhada (NPPP) e do sistema radicular (NPPSR), na parte aérea da cana-de-açúcar, aumentou com o tempo (Tabela 1). O aumento na recuperação pela cana-planta durante o seu ciclo pode ser atribuído à diminuição da relação $\mathrm{C}: \mathrm{N}$ inicial dos resíduos culturais, o que a teria deixado próxima à dos microrganismos do solo, intensificando a mineralização e disponibilizando mais $\mathrm{N}$ para a cultura (Basanta et al., 2003). Embora a estimativa do aproveitamento de $\mathrm{N}$ oriundo da mineralização de resíduos culturais tenha crescido expressivamente com o tempo, ele pouco contribuiu para a nutrição da cultura, pois ao final do ciclo apenas 3,1 e 2,2 $\mathrm{kg} \mathrm{ha}^{-1}$ de $\mathrm{N}$ proveniente dessas fontes foram acumulados, cerca de 6,0 e $6,5 \%$ do $\mathrm{N}$ total presente inicialmente na palhada e no sistema radicular, respectivamente. Essa estimativa se aproxima da verificada por $\mathrm{Ng}$ Kee Kwong et al. (1987), que obtiveram valores entre 5 e $10 \%$ de recuperação do $\mathrm{N}$ da palhada de cana-de-açúcar.

O NPPF, na dose aplicada de $80 \mathrm{~kg} \mathrm{ha}^{-1}$ de $\mathrm{N}$, foi cerca de três vezes superior ao $\mathrm{N}$ proveniente da palhada e do sistema radicular (Tabela 2). Esses resultados foram obtidos por medidas diretas de toda a

Tabela 2. Quantidades de N provenientes da ureia (NPPF), da palhada (NPPP) e do sistema radicular (NPPSR) nos compartimentos da cana-planta colmo, folha seca e ponteiro, e recuperação em cada compartimento (R) do $\mathrm{N}$ dessas fontes.

\begin{tabular}{|c|c|c|c|c|c|c|c|c|c|c|c|c|}
\hline \multirow[t]{2}{*}{ Fonte de ${ }^{15} \mathrm{~N}$} & \multicolumn{3}{|c|}{ Colmo } & \multicolumn{2}{|c|}{ Folha seca } & \multirow[b]{2}{*}{ R (\%) } & \multicolumn{3}{|c|}{ Ponteiro } & \multicolumn{3}{|c|}{ NTPA $^{(1)}$} \\
\hline & $(\%)$ & $\left(\mathrm{kg} \mathrm{ha}^{-1}\right)$ & $\mathrm{R}(\%)$ & $(\%)$ & $\left(\mathrm{kg} \mathrm{ha}^{-1}\right)$ & & $(\%)$ & $\left(\mathrm{kg} \mathrm{ha}^{-1}\right)$ & $\mathrm{R}(\%)$ & $(\%)$ & $\left(\mathrm{kg} \mathrm{ha}^{-1}\right)$ & $\mathrm{R}(\%)$ \\
\hline Jreia & $16,1 \pm 1,9$ & $18,4 \pm 2,1$ & $23,0 \pm 2,6$ & $15,9 \pm 2,0$ & $3,3 \pm 0,3$ & $4,1 \pm 0,4$ & $14,2 \pm 2,0$ & $2,6 \pm 0,7$ & $3,2 \pm 0,9$ & - & $24,3 \pm 3,1$ & $30,3 \pm 3,7$ \\
\hline Palhada & $5,5 \pm 2,4$ & $6,3 \pm 2,4$ & $12,2 \pm 4,5$ & $2,4 \pm 0,7$ & $0,5 \pm 0,2$ & $0,9 \pm 0,3$ & $2,3 \pm 1,2$ & $0,4 \pm 0,1$ & $0,8 \pm 0,2$ & - & $7,2 \pm 2,4$ & $13,9 \pm 4,5$ \\
\hline Sistema radicular & $1,3 \pm 0,3$ & $1,5 \pm 0,3$ & $4,5 \pm 0,9$ & $1,9 \pm 0,9$ & $0,4 \pm 0,1$ & $1,2 \pm 0,3$ & $1,2 \pm 0,2$ & $0,2 \pm 0,1$ & $0,7 \pm 0,2$ & - & $2,1 \pm 0,3$ & $6,4 \pm 0,9$ \\
\hline
\end{tabular}

${ }^{(1)}$ Média \pm desvio-padrão $(\mathrm{n}=4)$ do nitrogênio total da parte aérea. 
parte aérea da cana-planta. Na colheita, cerca de $75 \%$ de N-ureia acumulado na parte aérea estava nos colmos. Resultado semelhante foi obtido com o $\mathrm{N}$ proveniente dos resíduos culturais, em que a maior parte também estava contida nos colmos. Estes resultados podem ser explicados pela maior massa de matéria seca dos colmos em comparação aos demais compartimentos da planta, e têm como consequência maior acumulo de N-total (Franco et al., 2010).

A recuperação pela cana-planta do $\mathrm{N}$ do fertilizante (R) foi de 30,3\%, semelhante à obtida por Vitti (2003) em experimento com soqueira. Pôde-se observar que, dos $51,5 \mathrm{~kg} \mathrm{ha}^{-1}$ de $\mathrm{N}$ presentes na palhada, ao plantio da cana-de-açúcar, apenas 7,2 $\pm 2,4 \mathrm{~kg} \mathrm{ha}^{-1}$ de $\mathrm{N}$ foram acumulados pela cultura na parte aérea, na colheita, o que representou $13,9 \pm 4,5 \%$ do $\mathrm{N}$ da palhada. No sistema radicular ( $33 \mathrm{~kg} \mathrm{ha}^{-1}$ de $\mathrm{N}$ total), este valor foi de apenas $2,1 \pm 0,3 \mathrm{~kg} \mathrm{ha}^{-1}$ de $\mathrm{N}$, ou $6,4 \pm 0,9 \%$ do $\mathrm{N}$ total desse resíduo (Tabela 2).

A contribuição do $\mathrm{N}$ proveniente da palhada e do sistema radicular para a nutrição da cultura foi baixa, ou seja, representou apenas 4,7 e $1,4 \%$ do $\mathrm{N}$ total acumulado pela parte aérea da cana-planta, ao término de seu ciclo de crescimento de 18 meses. Isso pode ser explicado pela baixa decomposição desses resíduos, independentemente de sua incorporação parcial ao solo. É provável que a contribuição desses resíduos seja maior nas safras subsequentes, como mencionado por Vallis et al. (1996), que sugerem redução na adubação nitrogenada em razão do $\mathrm{N}$ disponibilizado pela palha, após um período de 20 anos em sistema sem queima. Em condições de campo, $\mathrm{Ng}$ Kee Kwong et al. (1987) concluíram que os resíduos de cana-de-açúcar representariam uma fonte de $\mathrm{N}$ pouco significativa para as plantas, considerando-se um estoque maior do que $3.000 \mathrm{~kg} \mathrm{ha}^{-1}$ de $\mathrm{N}$ no solo e de apenas $40 \mathrm{~kg} \mathrm{ha}^{-1}$ de resíduos.

\section{Conclusões}

1. $\mathrm{O}$ aproveitamento de $\mathrm{N}$ oriundo da mineralização de resíduos culturais da palhada e do sistema radicular aumenta expressivamente com o tempo, no entanto, esta fonte pouco contribui para a nutrição da cultura.

2. A cana-planta recupera $30,3 \pm 3,7 \%, 13,9 \pm 4,5 \%$ e $6,4 \pm 0,9 \%$ de ${ }^{15} \mathrm{~N}$-ureia, do ${ }^{15} \mathrm{~N}$-palhada e de ${ }^{15} \mathrm{~N}$-sistema radicular, respectivamente.
3. As contribuições de $\mathrm{N}$ proveniente da ureia, da palhada e do sistema radicular representam, respectivamente, $15,9 \%, 4,7 \%$ e $1,4 \%$ do $\mathrm{N}$ total acumulado pela parte aérea, na colheita da cultura.

\section{Agradecimentos}

Ao Conselho Nacional de Desenvolvimento Científico e Tecnológico e à Fundação de Amparo à Pesquisa do Estado de São Paulo, por concessão de bolsas e suporte financeiro; ao Centro de Tecnologia Canavieira e à Usina São Martinho, pelo apoio logístico.

\section{Referências}

BASANTA, M.V.; DOURADO-NETO, D.; REICHARDT, K.; BACCHI, O.O.S.; OLIVEIRA, J.C.M.; TRIVELIN, P.C.O.; TIMM, L.C.; TOMINAGA, T.T.; CORRECHEL, V.; CASSARO, F.A.M.; PIRES, L.F.; MACEDO, J.R. Management effects on nitrogen recovery in a sugarcane crop grown in Brazil. Geoderma, v.116, p.235-248, 2003.

BOLOGNA-CAMPBELL, I. Balanço de nitrogênio e enxofre no sistema solo-cana-de-açúcar no ciclo de cana-planta. 2007. 112p. (Doutorado) - Escola Superior de Agricultura Luiz de Queiroz, Piracicaba.

FARONI, C.E.; TRIVELIN, P.C.O.; SILVA, P.H. da; BOLOGNA, I.R.; VITTI, A.C.; FRANCO, H.C.J. Marcação de fitomassa de cana-de-açúcar com aplicação de solução de uréia marcada com ${ }^{15}$ N. Pesquisa Agropecuária Brasileira, v.42, p.851-857, 2007.

FENILLI, T.A.B.; REICHARDT, K.; TRIVELIN, P.C.O.; FAVARIN, J.L. Volatilization of ammonia derived from fertilizer and its reabsorption by coffee plants. Communications in Soil Science and Plant Analysis, v.38, p.1741-1751, 2007.

FRANCO, H.C.J.; OTTO, R.; FARONI, C.E.; VITTI, A.C.; OLIVEIRA, E.C.A.; TRIVELIN, P.C.O. Nitrogen in sugarcane derived from fertilizer under Brazilian field conditions. Field Crops Research, v.121, p.29-41, 2011.

FRANCO, H.C.J.; TRIVELIN, P.C.O.; FARONI, C.E.; VITTI, A.C.; OTTO, R. Stalk yield and technological attributes of planted cane as related to nitrogen fertilization. Scientia Agricola, v.67, p.579-590, 2010.

FRANCO, H.C.J.; VITTI, A.C.; FARONI, C.E.; CANTARELLA, H.; TRIVELIN, P.C.O. Estoque de nutrientes em resíduos culturais incorporados ao solo na reforma do canavial. STAB, v.25, p.249-256, 2007.

GALDOS, M.V.; CERRI. C.C.; CERRI, C.E.P. Soil carbon stocks under burned and unburned sugarcane in Brazil. Geoderma, v.153, p.347-352, 2009.

GAVA, G.J.C.; TRIVELIN, P.C.O.; VITTI, A.C.; OLIVEIRA, M.W. Recuperação do nitrogênio $\left({ }^{15} \mathrm{~N}\right)$ da uréia e da palhada por soqueira de cana-de-açúcar (Saccharum spp.). Revista Brasileira de Ciência do Solo, v.27, p.621-630, 2003. 
GUPTA, S.R.; SINGH, J.S. The effect of plant species, weather variables and chemical composition of plant material on decomposition in a tropical grassland. Plant and Soil, v.59, p.99-117, 1981.

JINGGUO, W.; BAKKEN, L.R. Competition for nitrogen during mineralization of plant residues in soil: microbial response to $\mathrm{C}$ and $\mathrm{N}$ availability. Soil Biology and Biochemistry, v.29, p.163-170, 1997.

MALAVOLTA, E.; VITTI, G.C.; OLIVEIRA, S.A. de. Avaliação do estado nutricional das plantas: princípios e aplicações. 2.ed. Piracicaba: Potafos, 1997. 319p.

MARTHA JÚNIOR, G.B.; TRIVELIN, P.C.O.; CORSI, M. Absorção foliar pelo capim-tanzânia da amônia volatilizada do ${ }^{15} \mathrm{~N}$-uréia aplicado ao solo. Revista Brasileira de Ciência do Solo, v.33, p.103-108, 2009.

MEIER, E.A.; THORBURN, P.J.; WEGENER, M.K.; BASFORD, K.E. The availability of nitrogen from sugarcane trash on contrasting soils in the wet tropics of North Queensland. Nutrient Cycling in Agroecosystems, v.75, p.101-114, 2006.

NEIRYNCK, J.; KOWALSKI, A.S.; CARRARA, A.; CEULEMANS, R. Driving forces for ammonia fluxes over mixed forest subjected to high deposition loads. Atmospheric Environment, v.39, p.5013-5024, 2005.

NG KEE KWONG, K.F.; DEVILLE, J. The course of fertilizer nitrogen uptake by rainfed sugarcane in Mauritius. The Journal of Agricultural Sciences, v.122, p.385-391, 1994.

NG KEE KWONG, K.F.; DEVILLE, J.; CAVALOT, P.C.; RIVIERE, V. Value of cane trash in nitrogen nutrition of sugarcane. Plant and Soil, v.102, p.79-83, 1987.

SANTOS, H.G. dos; JACOMINE, P.K.T.; ANJOS, L.H.C. dos; OLIVEIRA, V.A. de; OLIVEIRA, J.B. de; COELHO, M.R.; LUMBRERAS, J.F.; CUNHA, T.J.F. (Ed.). Sistema brasileiro de classificação de solos. 2.ed. Rio de Janeiro: Embrapa Solos, 2006. $306 \mathrm{p}$.
TRIVELIN, P.C.O.; OLIVEIRA, M.W. de; VITTI, A.C.; GAVA, G.J. de C.; BENDASSOLLI, J.A. Perdas de nitrogênio da uréia no sistema solo-planta em dois ciclos de cana-de-açúcar. Pesquisa Agropecuária Brasileira, v.37, p.193-201, 2002.

TRIVELIN, P.C.O.; VICTORIA, R.L.; RODRIGUES, J.C.S. Aproveitamento por soqueira de cana-de-açúcar de final de safra do nitrogênio da aquamônia- ${ }^{15} \mathrm{~N}$ e uréia- ${ }^{15} \mathrm{~N}$ aplicado ao solo em complemento à vinhaça. Pesquisa Agropecuária Brasileira, v.30, p.1375-1385, 1995.

VALLIS, I.; PARTON, W.J.; KEATING, B.A.; WOOD, A.W. Simulation of the effects of trash and $\mathrm{N}$ fertilizer management on soil organic matter levels and yields of sugarcane. Soil \& Tillage Research, v.38, p.115-132, 1996.

VITTI, A.C. Adubação nitrogenada da cana-de-açúcar (soqueira) colhida mecanicamente sem a queima prévia: manejo e efeito na produtividade. 2003. 114p. Tese (Doutorado) Universidade de São Paulo, Piracicaba.

VITTI, A.C.; FERREIRA, D.A.; FRANCO, H.C.J.; FORTES, C.; OTTO, R.; FARONI, C.E.; TRIVELIN, P.C.O. Utilisation of nitrogen from trash by sugarcane ratoons. International Sugar Journal, v.28, p.249-253, 2010.

VITTI, A.C.; FRANCO, H.C.J.; FARONI, C.E.; CANTARELLA, H.; TRIVELIN, P.C.O. Balanço de massas e de nutrientes da palhada e da rebrota de cana desseca com glifosato. STAB, v.25, p.30-33, 2007.

VITTI, A.C.; TRIVELIN, P.C.O.; CANTARELLA, H.; FRANCO, H.C.J.; FARONI, C.E.; OTTO, R.; TRIVELIN, M.O.; TOVAJAR, J.G. Mineralização da palhada e crescimento de raízes de cana-de-açúcar relacionados com a adubação nitrogenada de plantio. Revista Brasileira de Ciência do Solo, v. 32, p.2757-2762, 2008.

Recebido em 13 de dezembro de 2010 e aprovado em 14 de fevereiro de 2011 